EPJ Web of Conferences 73, 02008 (2014)

DOI: $10.1051 /$ epjconf/20147302008

(C) Owned by the authors, published by EDP Sciences, 2014

\title{
Di-hadron SIDIS measurements at CLAS
}

\author{
Silvia Pisano ${ }^{a}$ for the CLAS Collaboration \\ INFN - Istituto Nazionale di Fisica Nucleare, via Enrico Fermi, 40, 00044 Frascati, Rome, Italy
}

\begin{abstract}
Semi-inclusive deep-inelastic scattering (SIDIS) is an essential tool to probe nucleon internal structure. Through single hadron SIDIS processes, indeed, it is possible to access the TMDs, containing information on both the longitudinal and transverse motion of the partons. In recent years, moreover, an increasing attention has been devoted to dihadron SIDIS. It constitutes the golden channel to access the higher-twist collinear Parton Distribution Functions $e(x)$ and $h_{L}(x)$, so far only marginally known, whose extraction will complete the collinear description of the nucleon at the twist-3 level. The CLAS detector in the Hall-B at JLab, thanks to its large acceptance, is particularly suited for such measurements. Analyses aiming at the extraction of dihadron SIDIS Beam and TargetSpin Asymmetries are presently in progress. In these proceedings, preliminary results for the Beam-Spin Asymmetry are reported, together with a summary of the dihadron SIDIS experimental program at JLab.
\end{abstract}

\section{Introduction}

Despite many years of investigation, both theoretical and experimental, the collinear picture of the proton is still far form being complete. At the twist-3 level, the nucleon is described through six Parton Distribution Functions (PDFs), that depend on the fraction $x$ of the proton total momentum carried by the parton: the leading twist unpolarized $f_{1}(x)$, the helicity distribution $g_{1}(x)$ and the so-called transversity $h_{1}(x)$ and the three higher twist ones, $e(x), h_{L} x$ and $g_{T}(x)$ [1,2]. Among them, $f_{1}(x)$ and $g_{1}(x)$ are pretty well known, having being extracted through Inclusive Deep Inelastic Scattering experiments. $h_{1}(x)$, on the other hand, due to its chiral-odd nature, appears in observables only through the coupling with a second chiral-odd object, and can only be accessed through Semi-Inclusive DIS processes, where at least one hadron is detected in the final state together with the outgoing electron. In these processes, indeed, the tranversity distribution appears coupled to the Fragmentation Function, another chiral-odd object describing the hadronization of the struck quark that leads to the final state hadrons. As to the three higher-twist PDFs, $e(x)$ and $h_{L} x$ are almost unknown, while $g_{T}(x)$ has been also extracted through inclusive DIS measurements. These higher-twist PDFs encodes important information on the physics of the largely unexplored quark-gluon correlations. Furthermore, the $x$-integral of $e(x)$ is related to the poorly known scalar charge of the nucleon, a fundamental quantity sensible to parityviolating components of the nucleon-nucleon interactions, while the $x$-integral of $h_{1}(x)$ is connected to

\footnotetext{
ae-mail: silvia.pisano@lnf .infn.it
}

This is an Open Access article distributed under the terms of the Creative Commons Attribution License 4.0, which permits unrestricted use, distribution, and reproduction in any medium, provided the original work is properly cited. 
the nucleon tensor charge, so far only poorly constrained by the available data. The cleanest process to access the unknown PDFs is rapresented by the Di-hadron Semi-Inclusive Deep Inelastic Scattering (dihadron SIDIS), where, together with the outgoing electron, at least two hadrons are detected in the final state. The cross-section of such a process, indeed, is described in terms of specific Structure Functions (SF) [3, 4], where the PDFs of interest appear coupled to the so-called Interference Fragmentation Functions, i.e. functions spefically introduced to describe the hadronization of the struck quark into two hadrons. With respect to the single-hadron case, furthermore, the PDFs appear coupled to the FF only through a simple product, and not through a convolution over the quark tranverse momentum, making the actual extraction of the parton distribution from di-hadron SIDIS data fully model independent. The CEBAF Large Acceptance Spectrometer (CLAS) [5] installed in the Hall-B at the Thomas Jefferson Accelerator Facility, is particularly suited for the extraction of these quantities. Its large acceptance implies a good capability in the reconstruction of multi-particle final states, allowing the investigation of the aforementioned process in a wide range of kinematics.

\section{CLAS data sets}

The CLAS experiment performed two analysis devoted to the extraction of di-hadron SIDIS related observables. One has been performed on a data set collected in 2003 by impinging a $5.5 \mathrm{GeV}$ longitudinally-polarized electron beam - provided by the CEBAF - on an unpolarized liquid-hydrogen target ${ }^{2} \mathrm{H}$, for a total integrated luminosity of $21 \mathrm{fb}^{-1}$. The second data set has been collected in 2009 with a slightly larger electron energy $(5.967 \mathrm{GeV})$, and by exploiting a longitudinally-polarized ${ }^{3} \mathrm{~N} \mathrm{H}$ target. Due to the polarization degrees of freedom active in the process, the first data set allows only the extraction of the longitudinal Beam-Spin Asymmetry $A_{L U}$, while the presence of a polarized target in the second data set provides access also to the Target-Spin Asymmetry $A_{U L}$ and to the Double-Spin Asymmetry $A_{L L}$. In this proceedings, preliminary results for $A_{L U}$ will be shown, both on the ${ }^{2} H$ and the ${ }^{3} \mathrm{NH}$ target. Extraction of the Target and of the Double-Spin Asymmetries is in progress on the ${ }^{3} \mathrm{NH}$ data.

\section{Beam-spin asymmetry extraction}

$A_{L U}$ turns out to be proportional to the following structure function:

$$
F_{L U}^{\sin \phi_{R}}=-x \frac{|R| \sin \theta}{Q}\left[\frac{M}{M_{h}} x e^{q}(x) H_{1}^{\varangle q}\left(z, \cos \theta, M_{h}\right)+\frac{1}{z} f_{1}^{q}(x) \widetilde{G}^{\varangle q}\left(z, \cos \theta, M_{h}\right)\right]
$$

where $e(x)$ appears coupled to the Interference Fragmentation Function $H_{1}^{\varangle q}$, recently extracted [6] through the Belle data [7]. The channel of interest in these analyses is the semi-inclusive electroproduction of two hadrons, i.e. $e p \rightarrow e \pi^{+} \pi^{-} X$. The final di-hadron sample has been selected by identifying final states where an electron and at least one $\pi^{+}$and one $\pi^{-}$are present. When more than one positive or negative pion is present, all the possible combinations are considered and treated as possible candidate events. The right kinematic region is then selected by applying: $i$ ) standard DIS cuts: $Q^{2}>1 \mathrm{GeV}$ (where $Q^{2}$ is the photon virtuality), to be in the deep-inelastic scattering regime, and $W>2 \mathrm{GeV}$ (where $W$ is the centre-of-mass energy of the system composed of the proton and the virtual-photon), to avoid the resonance region; ii) a cut on the missing mass of the system $m_{X}>1.05 \mathrm{GeV}$, to eliminate contamination from exclusive channels; iii) the cut $x_{F}=2 \frac{p_{\perp}}{W}>0$, where $p_{\perp}$ is the longitudinal momentum of the final hadron in the $p-\gamma^{*}$ reference frame, to select hadrons actually coming from the quark fragmentation (current-fragmentation region, CFR) and not from the target. $A L U$ is then defined as

$$
A_{L U}=\frac{1}{P_{B}} \frac{N^{+}-N^{-}}{N^{+}+N^{-}}
$$




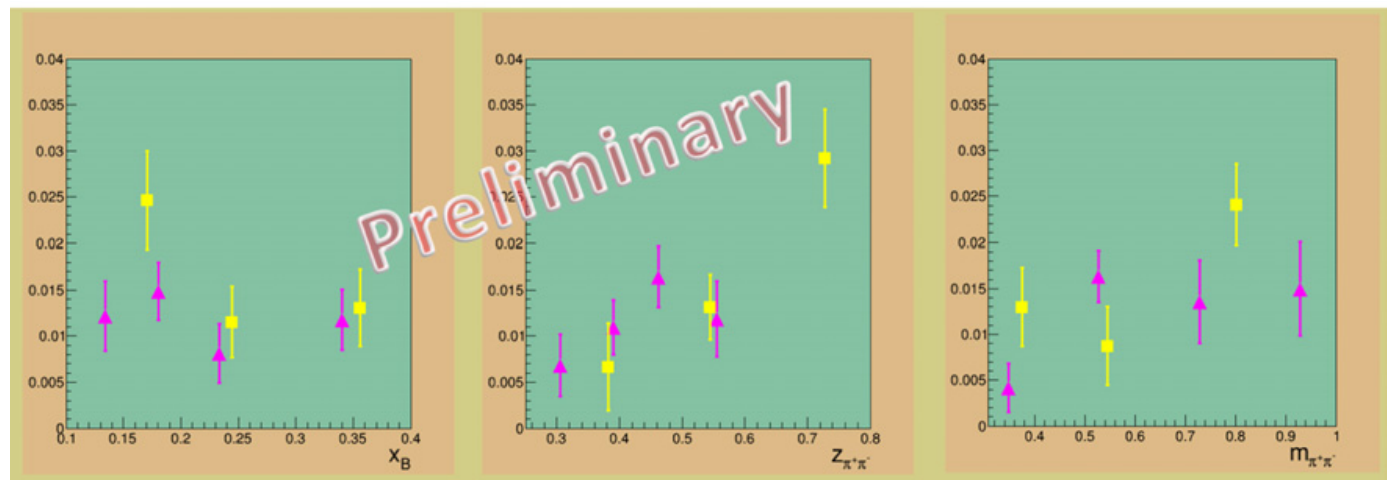

Figure 1. $A_{L U}$ dependence on $x_{B}$ (left plot), $z$ (middle plot) and $m_{\pi^{+} \pi^{-}}$(right plot), as extracted from the ${ }^{2} H$ data (yellow squares) and from the ${ }^{3} \mathrm{NH}$ data (magenta tringles).

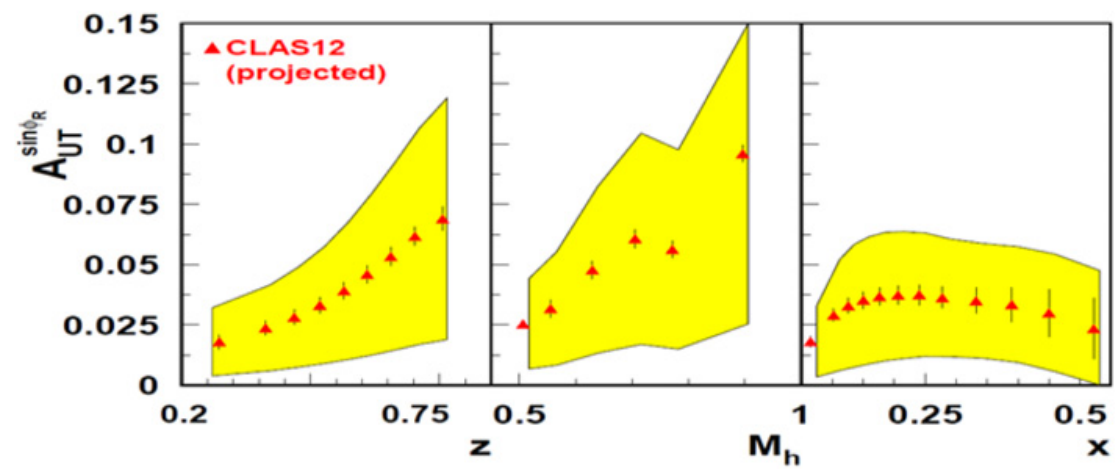

Figure 2. Projections for CLAS12 for the $A_{U T}$ dependence on $z$ (left plot), $m_{\pi^{+} \pi^{-}}$(middle plot) and $x_{B}$ (right plot). These projections corresponds to 110 days of data taking.

where $N^{ \pm}$represents the number of countings in the positive/negative helicity state, and $P_{B}$ is the beam polarization.

Both the ${ }^{2} \mathrm{H}$ and ${ }^{3} \mathrm{NH}$ data sets are divided in three sets of one-dimensional bins, in order to explore the dependence of the $\sin \phi$ moment of $A_{L U}\left(A_{L U}^{\sin \phi}\right)$ of $x_{B}$, the Bjorken variable, $m_{\pi^{+} \pi^{-}}$, the two-pion invariant mass, and $z$, the fraction of the virtual photon energy carried by the final hadron pairs. In Fig. 1 the results obtained on the two data sets are shown. $A_{L U}^{\sin \phi}$ turns out to be significanlty non zero in all the kinematic bins explored. Furthermore, the results from the two sets show consistent magnitude for the moments extracted, suggesting that no effect from the nuclear background present in the ${ }^{3} \mathrm{NH}$ target can be appreciated.

\section{Di-hadron in the $12-\mathrm{GeV}$ era}

The upgraded CEBAF will provide the four experimental halls an electron beam with an increased energy, that, for Hall-B, can arrive at $11 \mathrm{GeV}$. Hall-B equipment is presently being upgraded to a new detector, CLAS12, meant to perform high-acceptance, high-luminosity measurements. Furthermore, more targets will be available for CLAS12 operation. In particular, beyond the standard unpolarized and longitudinally-polarized targets already exploited in the $6 \mathrm{GeV}$ measurements, a tranversely-polarized target will be available. Di-hadron SIDIS is already part of the JLab12 physics program. In particular, the 
simultaneous presence of an unpolarized, a longitudinally-polarized and a tranversely-polarized target, operating with the longitudinally-polarized CEBAF electron beam, will allow to access the full set of Structure Functions describing the di-hadron SIDIS cross-section. Di-hadron SIDIS $A_{U T}$ is of particular interest, since it provides an access to the tranversity $h_{1}(x)$ complementary to the single-hadron one, and one proposal has been devoted to the extraction of this observables, whose projections are reported in Fig. 2. The projections show the statistical power of the CLAS12 data, that will be able to explore the $A_{U T}$ dependence on $x_{B}, z$ and $m_{\pi^{+} \pi^{-}}$in a wide kinematical range and with a high accuracy. It is important to stress here that CLAS12 will extend the constraint on $h_{1}(x)$ to the high-x (valence) region, where no data are presently available, that is also essential to constraint the nucleon tensor charge.

\section{Conclusions}

Di-hadron SIDIS processes constitute the easiest channel to access the leading-twist pdf $h_{1}(x)$ and the higher-twist pdfs $e(x), h_{L}(x)$. These last two PDFs can be accessed by extracting longitudinal Beam and Target-Spin Asymmetries. CLAS preliminary measurements show a significanlty non-zero $A_{L U}$ both on ${ }^{2} \mathrm{H}$ and ${ }^{3} \mathrm{NH}$. This extraction represents the first measurement of $A_{L U}$ and, combining the results presented here with the recently extracted $H_{1}^{\varangle q}$ [6] is possible, in principle, to get information on $e(x)$. Measurements of the Target and of the Double-Spin Asymmetries are also in progress, together with the extension of the analysis to channels with different pion combinations in the final state, e.g. $e p \rightarrow e \pi^{+} \pi^{0} X$. Di-hadron SIDIS is an important part of the $12 \mathrm{GeV}$ physics program of JLab, not only in the Hall-B, where measurements aiming at the extraction of the full set of Structure Functions for this process have been proposed, but also in Hall-A, where a letter of intent has been submitted by the SoLID Collaboration, proposing a measurement of $A_{U T}$ on a neutron target. The combination of the CLAS12 (proton) and SoLID (neutron) measurements will allow to operate a flavour separation, leading, eventually, to the extraction of $h_{1}(x)$ for the $u$ and $d$ flavours.

\section{References}

[1] Jaffe R.L. et al., Phys. Rev. Lett. 67, 552 (1991)

[2] Jaffe R.L. et al., Nucl. Phys. B 375, 527 (1992)

[3] A. Bacchetta, Ph. D. thesis, hep-ph/0212025

[4] A. Bacchetta et al., JHEP 02, 093 (2007), hep-ph/0611265

[5] B.A. Mecking et al., Nucl. Instr. Meth. A 503, 513 (2003)

[6] Courtoy, Aurore and Bacchetta, Alessandro and Radici, Marco and Bianconi, Andrea First extraction of interference fragmentation functions from $e^{+} e^{-}$data Phys. Rev. D 85, 114023 (2012)

[7] The BELLE Collaboration, A. Vossen et al., Phys. Rev. Lett. 107, 072004 (2011) (arXiv: 1104.2425) 